\title{
Schnelle Energiewende - bedroht durch Wutbürger und Umweltverbände? Protest, Beteiligung und politisches Risikopotenzial für Großprojekte im Kraftwerk- und Netzausbau
}

\author{
Marco Althaus
}

Zusammenfassung

Die deutsche Energiewende erfordert einen massiven Aus- und Neubau von Kraftwerken auf fossiler wie regenerativer Energiebasis sowie den Netzausbau. Die Beschleunigung der Planungs- und Genehmigungsverfahren hat für Regierung und Wirtschaft hohe Priorität. Dem stehen wachsende Beteiligungsansprüche von Bürgern und Interessengruppen gegenüber. Standortwahl und Umweltschutz führen oft zu Kontroversen und Protesten mit beträchtlichem politischem Risiko. Der Beitrag erörtert Widerstands- und Konfliktpotenzial im Umfeld von Energieprojekten und bewertet die Ressourcen und Strategien der Umweltverbände, deren Verhandlungsposition durch ein Urteil des Europäischen Gerichtshofs im Mai 2011 gestärkt wurde.

\section{Abstract}

Germany's about-face in energy policy, the »Energiewende«, requires massive extension and new construction of fossil and renewable fuel power plants, as well as grid and transmission line extension. Acceleration of planning and permission processes is a high priority for government and industry. It stands in contrast to growing citizens' and interest groups' demands for participation. Siting and environmental concerns often lead to controversies and protest with considerable political risk. The article discusses resistance and conflict potential in energy project environments and evaluates resources and strategies of environmental groups, whose negotiating position has been strengthened by a May 2011 European Court of Justice decision.

\section{Einführung}

Der Atomausstieg mag populär sein, die Anlagen, die die Nuklearmeiler ersetzen sollen, sind es nicht. Der Widerstand ist groß und wird wachsen, und er trifft in der Bevölkerung auf viel Verständnis. Das Tempo der Energiewende hängt aber am Tempo der Umsetzung von Kraftwerk- und Netzausbauprojekten. Politik und Wirtschaft setzen auf Beschleunigung. Diese ist schwer vereinbar mit wachsenden Ansprüchen an die Öffentlichkeitsbeteiligung. Zwei Akteure prägen die Ansprüche: lokale Bürgerinitiativen sowie professionell geführte Umweltverbände. Diese arbeiten in überregionalen Kampagnen zusammen. Das Urteil des Europäischen Gerichtshofs vom 12. Mai 2011 verhalf zudem der Verbandsklage im deutschen Recht zum Durchbruch. Damit wurden die Interventions- und Verhandlungsoptionen der Umweltverbände massiv gestärkt.
Der Beitrag erörtert anhand von Daten, Dokumenten und Literatur die Akzeptanzproblematik und das konflikthafte Projektumfeld, diskutiert Ressourcen- und Strategiefragen der Initiativen und Umweltverbände, und antwortet differenziert auf die Frage, ob sie die Energiewende bedrohen. Der Beitrag ist eine Erweiterung des Vortrags, den der Autor beim Kolloquium »Neuere Entwicklungen und Tendenzen im Wirtschaftsrecht und im Arbeitsrecht « des Fachbereichs Wirtschaft, Verwaltung und Recht am 23. Mai 2011 gehalten hat.

\section{Großprojekte für die Energiewende}

Als Reaktion auf die Katastrophe in den Kernreaktoren von Fukushima hat die Regierung Merkel die Energiewende ausgerufen. Der Ausstieg aus der Atomenergie wird forciert, die Energiebasis Deutschlands radikal verändert. Dies ist ein nationaler Kraftakt. In der Regie- 
rungserklärung im Juni 2011 sprach die Kanzlerin von einer »Herkulesaufgabe«: Lastenverteilungskonflikte, Risiken für die Klimaschutzziele, Gefahren für Industriearbeitsplätze, das mögliche »Steigen der Strompreise in das sozial nicht mehr Erträgliche« und andere Aspekte mehr belegen, warum sie sogar von einer »Quadratur des Kreises« spricht (Merkel 2011: 12963).

Technisch bedeutet die Energiewende den Ausbau alter konventioneller Kraftwerke sowie alter Kraftwerke der erneuerbaren Energieerzeugung (Wind, Photovoltaik, Solarthermie, Biomasse u. a.), den Neubau konventioneller und EE-Anlagen sowie den Aus- und Neubau von Übertragungs- und Verteilernetzen, insbesondere Höchst- und Hochspannungsleitungen. Die Schwächen der Stromnetze sind ein besonderer Brennpunkt. Überlastung und Zusammenbruch sind reale Szenarien, insbesondere weil der Ausbau von Windkraftanlagen in Norddeutschland und vor der Küste fernab der Verbrauchszentren die Schwerpunkte der Stromübertragung verlagert.

Die Energiewende führt zudem zur klimapolitisch ungewollten und äußerst unpopulären Renaissance der Stein- und Braunkohleverstromung. Sie wird die $\mathrm{CO}_{2}$ Emissionen deutlich erhöhen, wenn keine Ausgleichstechnologie eingesetzt wird. Eine solche wäre die $\mathrm{CO}_{2}$ Abscheidung und unterirdische Speicherung (Carbon Dioxide Capture and Storage, CCS). Pilotprojekte haben jedoch lokale Proteste ausgelöst, die Umweltverbände lehnen CCS ab.

Zwar war die Energiewende stets zentrale Forderung der organisierten Interessen des Umweltschutzes. Das heißt aber nicht, dass der Bau klimafreundlicher EE-Anlagen bedingungslos unterstützt wird. Umweltverbände legen auch an sie harte Kriterien an. Sie verlangen eine Beteiligung und Abwägung aller Interessen. Unmissverständlich formuliert Torsten Ehrke, Bundesvorsitzender der Grünen Liga: »Es kann nicht unser Anliegen sein, dass sich der notwendige Ausbau der erneuerbaren Energien zulasten von Natur und Umwelt vollzieht.« Die Balance müsse gehalten werden, »damit nicht der sogenannte Wutbürger später eingreifen muss« (Hörr 2011). Ähnlich ist es beim Netzausbau, um EE-Anlagen anzubinden. So fordert der Naturschutzbund, dass die "Auswirkungen auf Mensch, Umwelt und Natur minimiert« und unnötige Neubaumaßnahmen vermieden werden (NABU 2009).

Die andere Seite warnt vor zu viel Bedingungen und Beteiligung. »Künftig können nicht mehr jeder einzelne naturschutzrechtliche Belang und jedes Anwohnerin- teresse berücksichtigt werden «, schreiben von Daniels und Uibeleisen, Anwälte der politisch einflussreichen Wirtschaftskanzlei Freshfields, markig in der Financial Times Deutschland zu Offshore-Windparks und Stromtrassen. Die Bürgerproteste würden "paradoxerweise häufig angeführt von Atomkraftgegnern und erklärten Klimaschützern - ganz nach dem Motto: >Wasch mich, aber mach mich nicht nass . [...] Die öffentliche Anhörung in den Genehmigungsverfahren, die eigentlich der behördlichen Sachverhaltsermittlung und dem Interessenausgleich dienen soll, wird von den Projektgegnern häufig dazu zweckentfremdet, das Vorhaben mit Verfahrenstricks gezielt zu verzögern. [...] Die Erfahrungen der Praxis zeigen, dass öffentliche Anhörungen (sogenannte Erörterungstermine) in Wirklichkeit wenig zur Befriedung beitragen. Eher reißen sie die Gräben noch tiefer auf.« (Daniels et al. 2011)

Die scharfe Rhetorik gilt der bedrohlichen Protestlandschaft im Windsektor. Rund 70 Bürgerinitiativen agieren in Deutschland gegen Windkraft; eine ähnlich hohe Zahl von Initiativen findet sich im Bereich des Netzausbaus. Die Hälfte der Initiativen hat sich zwischen 2007 und 2009 gegründet (Becké 2011).

Aus- und Neubau von Kraftwerken und Netzen sowie der Einsatz umstrittener Technologien erzeugen wie alle Großprojekte unweigerlich einen Knoten politischer und rechtlicher Konflikte mit diversen Interessen- und Anspruchsgruppen (Stakeholder), die über viele Jahre hinweg in komplexen Prozessen der Kommunikation, Beteiligung und des Projektmanagements bearbeitet werden müssen.

Diese politischen Anforderungen sind kein lokal begrenztes Dilemma. Das zeigt eine für die Industrieverbände erstellte Allensbach-Umfrage (2011). Danach haben 76 Prozent der Bevölkerung generell Verständnis für Proteste gegen Großprojekte. 71 Prozent meinen, dass Bürger $\mathrm{zu}$ wenig informiert und eingebunden würden (ebd.: 3). 83 Prozent haben etwa beim Bau von Hochspannungsleitungen Verständnis für Proteste und Verhinderungsversuche, 76 Prozent selbst dann, wenn diese Leitung Ökostrom transportiert und von der Bürgermehrheit befürwortet wird. Ein ordnungsgemäßes Planungs- und Genehmigungsverfahren ist den meisten Bürgern nicht genug, um Großprojekte zu legitimieren. 68 Prozent akzeptieren Proteste gegen bereits genehmigte Bauprojekte. 62 Prozent stimmen der Aussage zu: »Wenn Entscheidungen getroffen werden, die die Mehrheit der Bürger für falsch hält, müssen solche Beschlüsse auch im Nachhinein korrigiert werden.« 
(ebd.: 7). Laut Allensbach wird der Nutzen großer Infrastrukturprojekte eher gering geschätzt. 64 Prozent halten die Energieinfrastruktur für gut oder sehr gut (ebd.: 4). 49 Prozent ist bewusst, dass mit der Energiewende größere Maßnahmen erforderlich werden. So gehen 85 Prozent von "großem Bedarf« bei erneuerbaren Energien aus. Nur 43 Prozent sehen ihn bei Energietrassen. Die Mehrheit sieht »wenig Bedarf « für neue konventionelle Kraftwerke (54 Prozent bei Gas-, 74 Prozent bei Kohlekraftwerken) (ebd: 5). In der Nähe des Wohnorts würden 79 Prozent »Versorgungsanlagen für erneuerbare Energien« befürworten (konkret nach Windparks u. a. wurde nicht gefragt). Aber die Mehrheit würde in der Heimat den Bau von Energietrassen (51 Prozent), Gas- (64) und Kohlekraftwerken (81) ablehnen (ebd.: 7). (IfD Allensbach 2011)

Nachbarschaftskonflikte sind bei Großprojekten alles andere als neu. Schwerwiegend ist nach Claus und Dames (2011), dass sie sich immer mehr zu Grundsatzfragen ausweiteten. Und: »[D]er Kern der Auseinandersetzung verschiebt sich. Während bislang Verwaltung und Politik für die Gewinnung von Akzeptanz für Industrieanlagen verantwortlich waren, sind nun auch Unternehmen selbst in der Pflicht, für Transparenz und gute Nachbarschaft zu sorgen« (2011). Energiewendeprojekte werden, wenn der Vorhabenträger das ignoriert, politisch unberechenbar. Sie tragen damit das Risiko des politischen Scheiterns oder der Verzögerung um viele Jahre.

\section{Beschleunigung, Bürokratie und Beteiligung}

Die Energiewende forciert alte Konflikte über die Beschleunigung von Großprojekten. Die Wirtschaft lobbyiert für mehr Tempo und schlankere Genehmigungsverfahren, um private wie öffentliche Investitionen frei zu setzen. Der Ruf wurde gehört: Anfangs ausgelöst durch den ostdeutschen Aufbaubedarf, wurden für Verkehrsprojekte Beschleunigungsgesetze verabschiedet, zuletzt das Infrastrukturplanungsbeschleunigungsgesetz (2006). In dieser Linie steht das Netzausbaubeschleunigungsgesetz (NABEG) von 2011. Entbürokratisierung und Beschleunigung sind wirtschaftspolitisch positiv besetzte Schlagworte. Sie sind aber Alarmsignal für Umweltverbände und Bürgerinitiativen. Denn lange Verfahren sichern ihnen Zugang. Mehr Tempo heißt dagegen,
- Verfahren schneller zum endgültigen Abschluss zu bringen und unumkehrbar zu machen;

- das Zeitfenster früher zu schließen, in dem Gegenargumente gesammelt und aufbereitet werden können;

- den Einsatz von Rechtsmitteln mit aufschiebender Wirkung zu beschneiden;

- weniger Spielraum für die Abwägung widerstreitender Belange, Interessenausgleich und Konsens;

- weniger Öffentlichkeit und weniger Transparenz.

Nach 20 Jahren Beschleunigungsgesetzen spricht Hubert Weiger, Vorsitzender des Umweltverbands BUND, von einem »Erosionsprozess der Bürgerbeteiligung « und »fortschreitender Entmündigung des Bürgers«. Er kritisiert insbesondere, dass Bürger nicht fortlaufend an Planungsverfahren beteiligt würden. Für Einwände seien ihnen enge Fristen gesetzt, der Projektträger könne seine Planung dagegen jederzeit ändern. Potenzial für mehr Tempo sieht er in früherer Beteiligung: »Je besser die Planung am Anfang ist, desto rascher kann sie realisiert werden.« Räumte man Bürgern mehr Rechte ein, nähme man den »Motzern« die Argumente (Becker 2011).

Einen »Mentalitätswandel bei der kommunikativen Begleitung von Großvorhaben « fordern auch Politikund Wirtschaftsberater wie Shah und Karten (2011), die am kontroversen Projekt der Fehmarnbeltquerung mitwirkten. Bürger wollten »rechtzeitig und ehrlich einbezogen werden und auf Augenhöhe mit den Verantwortlichen agieren« (ebd.: 46). Sie mahnen: »Es gibt keine rein technischen Großprojekte.« Kommunikation müsse von Anfang an und auch finanziell in die Gesamtstrategie integriert werden. Projektträger dürften Beteiligungsprozesse nicht als »Konsensmaschine« missverstehen. »Das Ziel lautet Vertrauen, nicht Konsens«, denn dafür lägen die Positionen oftmals zu weit auseinander. Kritiker sollten »darauf bauen können, dass die Projektträger kompetent und auch willens sind, ihre Anliegen ernst zu nehmen und konkrete Verbesserungsvorschläge zu berücksichtigen «.

Gesetzestreue allein reiche nicht aus, denn: „Gesetzliche Vorgaben gelten stets nur für den rechtlichen Planungsprozess. Starten die Planer eines Projekts die Kommunikation rund um ihr Bauvorhaben erst dann, wenn sie die Planfeststellungsunterlagen einreichen, ist es meist schon zu spät. Denn zu diesem Zeitpunkt sind Änderungen nur noch schwierig zu integrieren, und auch die Kosten dafür sind hoch. All das führt dazu, dass die Projektträger immer weniger bereit sind, sich 
auf die Wünsche betroffener Bürger und Umweltverbände einzulassen. Oft folgt daraus eine Konfrontation, die vor Gericht endet und viel Zeit und Geld kostet.« (2011: 47)

Stuttgart21 habe gezeigt, wie wichtig eine Kommunikation von Zwischenschritten sei, um die »böse Überraschung « der Bürger zu vermeiden. »Wenn hierzulande technische Pläne oder Ergebnisse veröffentlicht werden, dann in der Regel erst, wenn klar ist, dass sich daran nichts mehr ändern wird. In Skandinavien dagegen ist es üblich, dass Zwischenstände bei der Planung mit kommuniziert werden. Diese Praxis überrascht in Deutschland und erfordert einiges an Mut - vor allem, weil die Öffentlichkeit Veränderungen lieber als Korrektur vorheriger Fehler statt als natürlichen Teil einer optimierten Planung ansieht.« (Shah et al. 2011)

Frühe Beteiligung hält auch Stuttgart21-Schlichter Heiner Geißler für nötig. Das »obrigkeitsstaatliche Baurecht « stehe dem jedoch entgegen, es sei »Basta-Politik « (Wirner 2011). »In Deutschland läuft es doch immer so, dass am Anfang die Entscheidung steht, dann erst werden die Leute angehört [...] deswegen haben wir die Krawalle«, so Geißler (Bernard et al. 2011: 28).

Diese Kritik und die die Politik erschütternden Konflikte um Stuttgart21 konnte der Gesetzgeber 2011 beim Netzausbaubeschleunigungsgesetz (NABEG) nicht mehr ignorieren. Durchsetzbar war dieses für die Energiewende wichtige Gesetz nur, weil es hohe Anforderungen an Transparenz und Öffentlichkeitsbeteiligung stellt. »Die Lektion, dass die Bürger bei so weitreichenden Entscheidungen einzubinden sind, hat die Bundesregierung inzwischen gelernt«, stellt Mahnke (2011) fest. »Das NABEG stellt den Versuch dar, möglichst viel Beschleunigung im Netzausbau bei einer weitestgehenden Öffentlichkeitsbeteiligung zu erreichen. «

Berlin will dadurch »möglichst umfassende Transparenz herstellen. Ziel ist es, größere Akzeptanz für den Leitungsausbau zu erreichen und den Netzausbau so zu beschleunigen« (Bundesregierung 2011). Bürger werden äußerst früh in die Planung für einzelne Stromtrassen einbezogen, Beteiligung wird auch über das Internet ermöglicht, Interessenträger dürfen umfassende Dateneinsicht verlangen. Für die Erweiterung bestehender Trassen, die auch paralleler Neubau heißen kann, gilt aber ein »vereinfachtes Verfahren« ohne diese Möglichkeiten. Neue Streitfälle und Akzeptanzprobleme sind wahrscheinlich. Umweltverbände warnen davor, dass Beschleunigungseffekte durch Gerichtsprozesse darüber, ob ein konkreter Fall vereinfacht behandelt werden dürfe, wieder »aufgefressen « werden (Mahnke 2011).

Die Industrie weiß, dass der politische Wind sich gedreht hat und Beschleunigung nicht mit Verwaltungsmitteln um jeden Preis durchsetzbar ist. Der Bundesverband der deutschen Industrie (BDI), Bauverbände und andere warnen zwar wie früher vor Investitionsstau, Planungsunsicherheit, Technikfeindlichkeit und sinkender Wettbewerbsfähigkeit. Doch zeigt die BDIKampagne »Innovationsagenda « (ab August 2011), wie verunsichert die Industrie ist. Die Kampagne thematisiert den Mangel an Akzeptanz selbst und liefert auch das Stichwort Stuttgart21 dazu. Dafür wurde auch die erwähnte Allensbach-Umfrage beauftragt und publiziert. Grundsätzlich will der BDI zwar nicht am Primat der verbindlichen Rechtsentscheidung rütteln. Mit Blick auf Baden-Württemberg betont der BDI: »Eine Landtags- oder Kommunalwahl und wechselnde politische Mehrheiten dürfen nicht zur Auflösung einer Vereinbarung führen«(BDI 2011b). Doch betont der BDI, dass die Wirtschaft für »Akzeptanz« und »Transparenz« selbst verantwortlich ist: »Deutschland erlebt in jüngster Zeit einen verstärkten Trend zum bürgerschaftlichen Engagement. Politik und Wirtschaft müssen gemeinsam kommunikative Legitimationsprozesse initiieren - mit dem Ziel, in der Bevölkerung mehr Akzeptanz für Großprojekte zu gewinnen und Deutschland dauerhaft voranzubringen « (BDI 2011a: 2). Das betreffe explizit auch die Investitionen in erneuerbare Energien und Netzinfrastruktur, »um die politisch gewollte Energiewende Realität werden zu lassen«. Dafür sei ein »neuer Konsens« nötig (BDI 2011b).

Beteiligung und schnellere Planungsverfahren seien kein Widerspruch. »Die langwierigen Planungs- und Genehmigungsverfahren sind aus Sicht der Wirtschaft ein zentraler Grund für mangelnde Akzeptanz von Infrastrukturprojekten.« Bürger hätten ein Recht auf Information und Anhörung, doch finde dieser Prozess zu spät statt, sei deshalb auch zu teuer, ineffizient und bürgerfern. »Kürzere Genehmigungsphasen sind auch deshalb notwendig, weil sie für den Bürger leichter nachvollziehbar sind. Ein Projekt, bei dem sich alleine das Planfeststellungsverfahren über Jahre hinzieht, lässt sich am Ende des Verfahrens nur noch schwer vermitteln.« Damit nicht genug, thematisiert der BDI aktiv »neue Wege der Bürgerbeteiligung «, Formate unterschiedlicher »freiwilliger Dialogforen, die auch Vertreter von Bürgerinitiativen, Naturschutzverbänden, der Wirtschaft und der Kirche einbeziehen«. Ihre Stärke liege gerade darin, dass sie nicht Teil des gesetzlich vor- 
geschriebenen Verwaltungsverfahrens seien. Der BDI nennt Mediation, Planungsdialoge mit »ergebnisoffener Konsensfindung « sowie Internetangebote mit aktuellen Informationen und Beteiligungsmöglichkeiten (BDI: 2011c).

Insgesamt sind dies ungewöhnliche Signale, mit denen die Wirtschaft die öffentliche Meinung auf ihre Seite zu ziehen versucht. Sie scheinen im Vergleich zu früheren Positionen fast so radikal wie die Koalitionskehrtwende bei der Atomenergie. Solche Veränderungen sind selten in Spitzenverbänden wie dem BDI, der die komplizierte Meinungsbildung von 38 Branchenverbänden mit 100.000 Unternehmen moderiert. Umso bemerkenswerter ist, wenn die Industrie selbst den Scheinwerfer auf ihr Akzeptanzproblem richtet. Nun muss sie aber auch liefern.

\section{Wutbürger und »Nimby«}

Proteste gegen Energieanlagen waren fast 40 Jahre lang auf die Atomkraft konzentriert. Gleichwohl gab es stets auch Widerstände gegen andere Großprojekte, gegen Kraftwerke und Leitungen, außerdem besonders bei der Verkehrsinfrastruktur. Proteste richten sich gegen Flughäfen, Autobahnen, Brücken, Bahntrassen und Kanäle. Protestiert wird gegen Fabriken und Industriegebiete, Müllverbrennungsanlagen und -deponien, gegen Freizeitparks, Factory-Outlet-Zentren, Hochhäuser, Gefängnisse oder Asylbewerberheime, Mobilfunkmasten oder McDonald's-Restaurants. Oder in München gegen die Olympiabewerbung (»NOlympia«).

Die breite Forschungsliteratur zu den sozialen Bewegungen, die ab den 1970er Jahre entstanden, erklärt die neueren Protestphänomene nur unzureichend. Politik, Wirtschaft und Wissenschaft sind gleichermaßen irritiert über die Radikalisierung einer bisher protestfernen, staatstragenden, bürgerlichen Mitte. In diese Deutungslücke stößt der Begriff »Wutbürger«. In seinem vielbeachteten Spiegel-Essay erklärt Kurbjuweit (2011: 26) das Protestphänomen durch »Zukunftsvergessenheit « einer alternden Gesellschaft. Das »Verhältnis von denen, die viel vom Wandel haben, und denen, die wenig davon haben, wird immer ungünstiger für eine dynamische Entwicklung des Landes«, meint Kurbjuweit. Der Wutbürger denke nur an sich und die Gegenwart: »Deshalb beginnt sein Protest in dem Moment, da das Bauen beginnt, also die Unannehmlichkeit. Nun schiebt er das beiseite, was Bürgertum immer ausgemacht hat: Ver- antwortlichkeit, nicht nur das Eigene und das Jetzt im Blick zu haben, sondern auch das Allgemeine und das Morgen. [...] Der Wutbürger hat das Gefühl, Mehrheit zu sein und die Lage besser beurteilen zu können als die Politik. Er macht sich zur letzten Instanz und hebelt dabei das gesamte System aus. Er versteht nicht oder will nicht verstehen, dass ein Sieg der Gegner von Stuttgart 21 jeden anderen Protest in Deutschland beflügelt. Fast jedes neue Kraftwerk, fast jede Hochspannungsleitung, fast jedes Windrad, fast jede Straße ist umstritten, weil sie nicht in Lebensgefühle passen oder Lebenslagen verändern.«(Kurbjuweit 2011)

Weitgehend bestätigt wird die journalistische Deutung durch das Göttinger Institut für Demokratieforschung. Es hat im Sommer 2011 am Protest gegen Stuttgart21 und den Flughafen Berlin-Brandenburg Beteiligte, Vertreter von 52 Initiativen gegen Windräder und Freileitungen sowie die Teilnehmer einer Großdemonstration »Pro Erdkabel« in Hannover gegen Stromleitungen interviewt. Fazit der 2.000 Stellungnahmen: 70 Prozent der Befragten sind über 45 Jahre alt, 16- bis 25-Jährige stellen nur ein Prozent. »Heute beherrschen die jungen Rentner - damals [in den 1970ern] so gut wie gar nicht präsent - das Bild des Bürgeraufbegehrens« (Walter 2011). Fast 60 Prozent der Aktivisten hätten einen Hochschulabschluss. Sie seien weit überwiegend mit ihren materiellen Lebensumständen zufrieden, unzufrieden aber mit Politik und Parteien. Sie wollten als Betroffene politische Einflusskanäle wirksam nutzen: »Denn natürlich sind die Handlungsmotive der Bürgerproteste nicht rundum selbstlos. Sie werden nicht allein von der Sorge um den Bestand der Fledermäuse, rarer Biotope oder uralter Bäume angetrieben. Die umtriebigen Wortführer gegen Flughafenausbau, Windräder und Oberleitungen sind in bemerkenswert großem Umfang (über 90 Prozent) Grundstückseigentümer und Hausbesitzer. Sehr prosaisch formuliert: Die Immobilienwerte stehen auf dem Spiel, wenn Stromleitungen und bis zu 150 Meter hohe Windräder in einem bis dahin beschaulichen Kurort den Blick auf eine CasparDavid-Friedrich-Landschaft verstellen« (Walter 2011).

Auffallend sei auch die recht hohe Quote an Unternehmern und Selbstständigen. Der Ingenieur im Ruhestand sei ebenfalls typisch: Er sei nicht fortschrittsfeindlich, argumentiere aber mit technischem Wissen, um Stromtransporttechniken, die sein Umfeld bedrohen, zu verhindern (Walter 2011). Das Beispiel der Bürgerinitiativen gegen Windkraftanlagen und Stromtrassen zeige allerdings, dass die Wutbürger-Klischees 
nicht immer zuträfen, hält Becké (2011) fest. Die Initiativen agierten meist »äußerst maßvoll«, in »ruhigen Formen des Protestes « sowie "produktiver Vernetzung und Kontaktpflege mit Vertretern aus Politik und Wirtschaft «. Statt Demonstrationen gebe es Mahnwachen, Lichterketten und Unterschriftenaktionen. Sie bemühten sich, komplexe Themen für die breite Öffentlichkeit zugänglich zu machen. Becké meint, die bürgerliche Mitte habe »keineswegs jedes Maß verloren«, ihre Ziele seien "größtenteils konstruktiv und vor allem lokal begrenzt, und ihr Empörungspotenzial scheint eher gering.«Sie überschätzten sich aber: »Sie schreiben sich ein extrem hohes Bedeutungsmaß zu und sind überzeugt, als unabhängiger und objektiver Informationslieferant an einer wichtigen Schaltstelle zwischen Politik und Wirtschaft zu sitzen. Je kleiner dabei die Initiative ist, desto höher wird auch der eigene Einfluss bewertet.«(2011)

Ein Teil der Protestphänomene lässt sich mit dem Sankt-Florians-Prinzip verbinden (»Heiliger Sankt Florian, verschon mein Haus, zünd andre an«). Die Grundhaltung zu Großprojekten ist: Allgemein ja, aber nicht hier und nicht auf meine Kosten. International wird vom Nimby-Syndrom gesprochen, als Akronym für »Not in my back yard.« Dazu ist vor allem in den USA eine veritable Nischenliteratur entstanden, einschließlich Büchern spezialisierter Berater, wie man in einer von »Nimbyismus « geprägten Lokalpolitik navigieren (Thomsett 2004), mit welchen Kampagnentechniken man »Nimby-Kriege« führen (Saint et al. 2009) oder »den Nimby-Drachen erlegen « soll (Inhaber 1998). Über das US-Phänomen fasste bereits Glaberson (1988) das zusammen, was in den jüngsten deutschen WutbürgerAnalysen gesagt wird. Die Nimby-Bewegung bestehe nicht aus Eiferern, sondern aus einer wachsenden Zahl von Lehrern, Vorstadtangestellten und Unternehmern, die in Kommunalgremien oder Bürgerversammlungen auftauchten und sich zeitlich begrenzt organisierten: "Nimbys are noisy. Nimbys are powerful. Nimbys are everywhere. Nimbys are people who live near enough to corporate or government projects - and are upset enough about them - to work to stop, stall or shrink them. Nimbys organize, march, sue and petition to block the developers they think are threatening them. They twist the arms of politicians and they learn how to influence regulators. They fight fiercely and then, win or lose, they vanish.«(Glaberson 1988)

Glaberson stellte fest, die Bürger wollten Wachstum, aber nur abstrakt, nicht in ihrer Nähe. Er bezog sich auf das aus der ökonomischen Theorie bekannte Dilemma konzentrierter Kosten und diffusen Nutzens: Die Nachteile für die unmittelbaren Nachbarn sind groß, der direkte Nutzen aber klein. Die Frage sei, ob Tausende kleiner Nimby-Siege noch Platz lassen für jene Anlagen, die der Wirtschaft und Allgemeinheit gemeinsam zugute kämen.

Die Motivationslage der Aktivisten entscheidet darüber, mit wem ein Vorhabenträger sinnvoll einen berechenbaren Dialog mit Erfolgsaussicht führen kann. Die Nimby-Haltung »nur bitte nicht hier« ist keine Totalopposition. Gruppen, die den Projektbedarf nicht grundsätzlich bestreiten, mögen nicht immer konstruktiv sein, aber sind kompromissfähig, wenn sie ernst genommen werden, wenn sie ein Stück Kontrolle über das Verfahren gewinnen und Anpassungen möglich sind. Anders sieht es aus, wenn Gruppen eine kompromisslose, ideologische Haltung einnehmen und ihr Ziel nicht die Beilegung, sondern die Eskalation der Konflikte ist. Entweder sie verweigern sich oder sie kapern und sabotieren Verhandlungen, Mediationen und Beteiligungsverfahren. Im ersten Fall kann man von Verhandlungsdemokratie sprechen, im zweiten von Verhinderungsdemokratie. Für Politiker, die in dieser Gemengelage als Vermittler oder ehrlicher Makler einen Interessenausgleich herbeiführen und befrieden möchten, ist die Radikalisierung und Polarisierung riskant: Sie werden stark unter Druck gesetzt, um sich sofort dafür oder dagegen auszusprechen. In diesem Freund-Feind-Schema gibt es keine Mittelposition.

\section{5 Überregionale Kampagnen}

Naturgemäß haben Bürgerinitiativen kleine Reichweiten. Lockere Strukturen und ein enger Fokus als Ein-Punkt-Gruppen unterscheiden sie von Umweltverbänden. Kommunikation und Interessenvertretung können koordiniert, kaum aber zentral und hierarchisch gesteuert werden. Eine engere Kooperation entsteht eher auf Landesebene, etwa bei Windkraftgegnern oder Erdkabel-Initiativen, die sich gegen Freileitungen wenden.

Dennoch sind Bürgerinitiativen fähig, sogar internationale Netzwerke zu bilden. So zählt die European Platform Against Windfarms (EPAW 2011) 500 Unterzeichnerorganisationen aus 22 Ländern, darunter 72 deutsche (u. a. Bundesverband Landschaftsschutz, Gegenwind). In Totalopposition will die EPAW den 
»Kampf gegen die Errichtung industrieller Windkraftanlagen« führen. Ihre Position ist, dass »diese unstete und unkontrollierbare Energie kein einziges Problem der Menschheit löst« und »Windkraftanlagen den Menschen, der Wirtschaft, den Staatsfinanzen und der Umwelt nichts anderes als beträchtliche Schäden zufügen $«($ EPAW 2011).

Auch wenn etwa die Anti-Windkraft-Initiativen ein erhebliches Medienecho erhielten, kam es bisher nicht zu bedeutenden überregionalen Kampagnen gegen regenerative Energien. Eine andere Dimension erreicht die Opposition gegen den Aus- und Neubau von Kohlekraftwerken. Hier sind wohlorganisierte und gut finanzierte Kampagnen zu beobachten, die lokale Initiativen mit den Stärken professionell geführter Umweltverbände verkoppeln und bundesweit einen Kampf um die politische Agenda und die öffentliche Meinung führen - mit Erfolg, wie man an der Allensbach-Umfrage ablesen kann. Energiekonzerne werden ebenso belagert wie Stadtwerke.

Führende Umweltorganisationen wie BUND und NABU, Greenpeace oder Robin Wood arbeiten systematisch und kampagnenförmig gegen Kohlekraftwerke. Die Kampagnen haben das Ziel, lokale Großprojekte zu verhindern, zugleich aber das Ziel, eine national konsequente Klimaschutzpolitik durchzusetzen. Zusätzliche Wirkung erreichen sie durch ihre Bündnispolitik in der Klima-Allianz Deutschland. 2007 zunächst für zwei Jahre gegründet, setzte sie ihre Arbeit nach der Bundestagswahl 2009 und zum Kopenhagener UN-Klimagipfel fort. Sie hat über 110 Mitglieder. Die mit zwei Hauptamtlichen besetzte Geschäftsstelle ist beim Forum Umwelt \& Entwicklung in Berlin angesiedelt. Die wesentlichen Ressourcen liegen bei den Mitgliedern. Dazu gehören Umweltverbände (BUND, NABU, WWF), Wirtschaftsverbände erneuerbarer Energien, Verbraucher- und Jugendverbände, Gewerkschaften (IG BAU), Kirchen, Hilfsorganisationen (Oxfam, Brot für die Welt, Misereor). Die Allianz vertritt so 10 Millionen Menschen. Die Breite soll signalisieren, dass die Anliegen »aus der Mitte der Gesellschaft« kommen. Die Allianz finanziert sich aus Mitgliedsbeiträgen (400 - 10.000 Euro) und Zuschüssen etwa der European Climate Foundation. Schwerpunkte sind "politische Lobbyarbeit, das Anstoßen und Organisieren von Debatten zur aktuellen Energie- und Klimapolitik und gemeinsame Veranstaltungen und Aktionen «, etwa Klima-Aktionstage und der Alternative Energiegipfel (Klima-Allianz Deutschland 2010).
Der Kern ist seit 2008 die Anti-Kohle-Kampagne, die auf ein Moratorium für Kohlekraftwerkpläne hinwirkt. Die Kampagne betreibt die Internetpräsenzen kohleprotest.de, stadtwerke-kohlefrei.de und keine-kohlefuer-kohle.de. Sie kooperiert mit der erfolgreichen Online-Kampagnenplattform Campact.de. Großes Medienecho hatte die bundesweite »Kohlosaurus «-Aktionstournee, bei der ein fünf Meter großer, schwarze Luftballons ausstoßender Kraftwerkturm mit Dinosaurierkopf und -pranken durch Kohlekraftwerkstandorte reiste. Für die Mitglieder stellt die Allianz Positions- und Hintergrundpapiere, Studien, Präsentationen, Flyer, Argumente, Vorlagen und Kampagnenmaterialien zur Verfügung. Mit ihrer zentralen Kampagnenlogistik und Pressearbeit multipliziert und verstärkt die Allianz die Stimmen örtlicher Initiativen. Detailliert informiert die Kampagne online über die derzeit zehn geplanten, zehn genehmigten oder im Bau befindlichen Kraftwerke sowie über die 17 verhinderten oder zurückgestellten Projekte. Nicht nur Baustopps werden als Erfolge präsentiert (z. B. beim EON-Kraftwerk Datteln), sondern auch Projektänderungen: So schreibt sich die Kampagne den Verdienst zu, Vattenfall zum Verzicht auf ein Kohlekraftwerk in Berlin zugunsten eines Biomasseund Gasprojekts getrieben und das Stadtwerke-Konsortium Trianel zum Verzicht auf ein Kohlekraftwerk in Krefeld bewegt zu haben, wo nun ein Gas- und Dampfturbinenkraftwerk errichtet werden soll (Klima-Allianz Deutschland 2011).

Doch schreibt die Allianz nicht nur eine Erfolgsstory. Unzufrieden mit schwach besuchten Demonstrationen, erwog der BUND einen Ausstieg. »Zudem empfanden einige Mitglieder die Klima-Allianz offenbar als Konkurrenz zum eigenen Verband «, konstatiert Kreutzfeld (2010). Eine Strategiedebatte setzte ein, in der vor »ritualisiertem Aktionismus « und dem »Schmoren im eigenen Saft « gewarnt wurde. Das Bündnis müsse zum "politischen Faktor« werden und Lobbying bei Entscheidungsträgern der deutschen Energiepolitik betreiben, hieß es aus dem Lager der Umweltverbände. Entwicklungsverbände sahen das anders: Das Bündnis solle »weiterhin als breit angelegter >Verstärker « wirken « und auf den internationalen Klimaschutz orientiert sein. Die Befürworter nationaler, energiepolitisch fokussierter Lobbyarbeit setzten sich durch. Sie planen mehr Personal, höhere Mitgliedsbeiträge und eine Anhebung des Jahresetats auf 500.000 Euro (Kreutzfeldt 2010).

Die Klima-Allianz ist nur begrenzt ein strategisches Zentrum. Breite ist nicht gleich Schlagkraft, sondern be- 
lastet Handlungs- und Strategiefähigkeit, Bindewirkung und Koordinationskraft. Sie spiegelt die in der Szene der Nichtregierungsorganisationen starken Fragmentierungsprozesse, Konflikte zwischen ressourcenstarken Profiverbänden und lockeren Grassroots-Initiativen, widersprüchlichen Strategieansätzen und die Tendenz, sich untereinander abzugrenzen und wieder »auf eigene Rechnung« zu arbeiten (Unmüßig 2011) wider.

\section{Verbandsklage: Schlüsselinstrument der Umweltverbände?}

Umweltverbände haben ein Portfolio politischer Instrumente an der Hand. Ob und wie sie zum Einsatz kommen, hängt von Strategie, Mitgliederbasis, Finanzen, hauptamtlichem Personal, Freiwilligenmanagement, Erfahrung und Vernetzung ab. Zu den Instrumenten zählen

- Medien- und Öffentlichkeitsarbeit einschließlich Aktionen und Kampagnen;

- Praktische Projekte (insbesondere im Naturschutz) und wissenschaftliche Studien;

- Bündnisse und Kooperationen mit anderen Organisationen, durchaus auch der Wirtschaft;

- Einwirkung auf und Mitarbeit in Wahlkämpfen;

- Stakeholder-Verhandlungen, in denen die Verbände bei Entscheidungen und Projekten auf freiwilliger Basis einbezogen werden, etwa von Unternehmen oder bei der informellen Bürgerbeteiligung durch Behörden;

- Lobbying, d. h. die direkte Einflussnahme auf Entscheidungsträger in (a) Gesetzgebungsverfahren und (b) bei der Rechtsumsetzung durch die Verwaltung; und

- gerichtliche Prozessführung.

Recht ist sowohl Gegenstand als auch Instrument der Lobbyarbeit. Die umweltrechtliche Kompetenz wird u. a. eingesetzt durch Hausjuristen als Sachverständige in Prozessen oder bei der Politik, durch Gerichtsverfahren begleitende Öffentlichkeitsarbeit (Litigation-PR), durch Unterstützung von Musterprozessen und durch Klagen als Betroffene, wenn der Verband etwa selbst ein Grundstück kauft. Verbände können Informationsfreiheit- und Umweltinformationsgesetze ebenso nutzen wie Informations- und Beteiligungsrechte im Planungsund Baurecht. Teils werden sie wie Träger öffentlicher Belange behandelt. So gewonnene Informationen helfen den Einsatz von Rechtsmitteln vorzubereiten. »Sie beschaffen sich von Vorhabenträgern Informationen nur zu dem Zweck, sie in Klagen zu verwenden«, kritisiert etwa der Wirtschaftsanwalt von Daniels (WIT/ DIPA 2005).

Der politische Streit um die Verbandsklage wird seit über 30 Jahren geführt. Befürworter halten die Stellvertreterfunktion für alternativlos, da die Umwelt sonst keinen Anwalt habe (»fish can’t go to court«). Sie argumentieren mit dem Wert der Demokratisierung und Bürgerbeteiligung sowie dem Vollzugsdefizit: Damit ist gemeint, dass Gesetze zwar beschlossen, aber von der Verwaltung wegen Mangel an Personal und Willen nicht umgesetzt werden. "So steht die Umweltverwaltung insbesondere bei größeren Vorhaben unter einem erheblichen politischen Druck, eine einmal projektierte Anlage zu genehmigen und durch Auflagen und Kontrollen nicht zu belasten «, begründet Wegener (2011: 364). Zugespitzt heißt es: »Ohne die Möglichkeit der Verbandsklage stünden weite Teile des Umweltrechts bald nur noch auf dem Papier « (Rath 2006: 6). Die Befürworter nennen die Verbandsklage eine Hilfe für »ein Verhandeln auf gleicher Augenhöhe« (Remmel 2006). Festzuhalten ist, dass das Verbandsklagerecht funktional zum politischen Korporatismus passt.

$\mathrm{Zu}$ den Gegnern der Verbandsklage gehörten stets Vertreter der Verwaltungsgerichte und Wirtschaftsverbände (Koch 2007: 371). Sie bestreiten die demokratische Legitimation der Umweltverbände, kritisieren den Bruch mit der Rechtstradition des Individualrechtsschutzes, warnen vor Instrumentalisierung und Überlastung der Gerichte. Auch wird kritisiert, dass Umweltverbände öffentliche Fördermittel erhielten, die indirekt Prozesse finanzieren. Schließlich schade die Verbandsklage dem Investitionsstandort. Das seien »gut gepflegte Vorurteile«, befindet Koch (2007: 376). Die Erfahrungen - auch im Ausland - lieferten »keine tragfähige Bestätigung für die vielfältigen Befürchtungen von Prozessfluten und anderen Dysfunktionalitäten im Rechtssystem « (ebd.: 376). Empirisch weist Schmidt (2008) in einer Studie über die naturschutzrechtliche Klagetätigkeit 2002 - 2006 (138 Klagen) nach, »dass die Naturschutzverbände ihre Klagerechte regelmäßig nur in ausgewählten Fällen mit guten Erfolgsaussichten nutzen und damit die Verbandsklage - ihrem Zweck entsprechend - gezielt als Instrument zum Abbau von Vollzugsdefiziten im Naturschutzrecht einsetzen « (ebd.: 545). Ihre Erfolgsquote liege bei 40 Prozent. An Verwaltungsgerichtsverfahren zu Großprojekten habe die Verbandsklage aber nur 0,02 Prozent Anteil. »Es 
spricht daher viel dafür, dass die Realisierbarkeit von Infrastrukturvorhaben in Deutschland nicht ernsthaft in Frage steht « (ebd.: 553).

In der Rechtspraxis wirkt die Verbandsklage wenig dramatisch. Die kollektive Rechtsdurchsetzung, in der Verbände als Stellvertreter für Allgemeininteressen auftreten, ist im Naturschutzrecht von Bund und Ländern verankert (seit 2002 im Bundesnaturschutzgesetz, BNatSchG). Voraussetzung ist die staatliche Anerkennung als Naturschutzverband, was als Nadelöhr gilt. In den Ländern ist das Verbandsklagerecht teilweise ausgeweitet worden. Mit dem Umwelt-Rechtsbehelfsgesetz (UmwRG 2006) des Bundes wurde eine EU-Richtlinie (2003/35/EG) umgesetzt, die wiederum auf der AarhusKonvention basiert, die die Öffentlichkeitsbeteiligung und den Zugang zu Gerichten für Bürger und Umweltverbände erweiterte. Die Verbände erhielten ein Klagerecht, soweit sie damit Individualinteressen mit vertraten - es enthielt also keine Befugnis, altruistisch »für die Umwelt «zu klagen. Dennoch war es eine Option bei Genehmigungsverfahren für Industrie- und Infrastrukturprojekte. Die Wirtschaft befürchtete 2005/6 eine Klageflut, die aber nicht eintrat.

Die Verbandsklage blieb »Fremdkörper« im Recht, was sich in restriktiven Zulässigkeitsanforderungen und in beschränkter gerichtlicher Kontrolldichte widerspiegelte (Schlacke 2004). Andere Instrumente erschienen daher effizienter, also Öffentlichkeitsarbeit, Verhandlungen, Lobbying und Politikberatung als Sachverständige. Schon Anfang des Jahrzehnts war klar: Das Verbandsklagerecht ist kein Universalwerkzeug. Es hat eher die Wirkung »juristischer Nadelstiche« (Börnecke 2003). Fischer (2011) stellt heraus, dass die Umweltverbände weitgehend nur die Möglichkeit hatten, quasi als Nebenkläger die Rechte einzelner Betroffener mit zu vertreten: »Das führte zum einen dazu, dass Umweltgefahren nicht abhängig von ihrer Schwere in Verfahren Eingang fanden. Zentraler Prüfstein war das Vorhandensein einer subjektiven Betroffenheit, die tatsächlich schwerwiegenden öffentlichen Interessen blieben oftmals außen vor. Zudem verpflichtete es die Verbände, mit Einzelklägern zu paktieren, auch wenn man selbst in den Verbänden deren Motiven skeptisch gegenüber stand.« (Fischer 2011)

Als der BUND 2009 gegen das Kohlekraftwerk in Lünen klagte, wurde ihm zunächst die Klagebefugnis abgesprochen (BUND 2011b). Der BUND focht dies an und erreichte am 12. Mai 2011 ein Urteil des Europäischen Gerichtshofs, das Deutschland zur Änderung des
UmwRG verpflichtet, weil die Beschränkungen gegen EU-Recht und insbesondere gegen den Aarhus-Grundsatz des »weiten Zugangs zu Gerichten« verstoßen (EuGH, Rechtssache C-115/09). Mit sofortiger Wirkung können sich Umweltverbände bei Verbandsklagen etwa gegen alle Energieprojekte, die eine Umweltverträglichkeitsprüfung erfordern, direkt auf das Unionsrecht berufen.

Das Urteil hat viel Aufsehen erregt. Der BUND sieht das Urteil als »Meilenstein für mehr Bürgerbeteiligung und die Stärkung der Gemeinwohlinteressen bei industriellen Großvorhaben«(BUND 2011a). Der EuGH habe »der Verbandsklage endgültig zum Durchbruch verholfen «, meint Schoppen (2011: 2), »für Genehmigungen besteht deshalb ein erhöhtes Klagerisiko und weniger Planungssicherheit für Projekte.«Müller und Hofmann (2011) sagen voraus: »Die massive Stärkung des Klagerechts der Umweltverbände dürfte großen Einfluss auf die zukünftige Genehmigungs- und Klagepraxis bei der Realisierung von umweltsensiblen Großanlagen haben.«

Vor dem EuGH argumentierte der Bund, Deutschland habe auch ohne Verbandsklage eine große materielle Kontrolldichte im Umweltrecht, ein hohes Schutzniveau für Betroffene. Das griff die EuGH-Generalanwältin im Schlussantrag auf: »Ebenso wie ein Ferrari mit verschlossenen Türen hilft jedoch eine intensive Kontrolldichte in der Praxis wenig, wenn das System als solches für bestimmte Kategorien von Klagen nicht zugänglich ist« (Sharpston 2011). Nach dem Urteil kommentierte Appel (2011), der EuGH habe »den Verbänden den Ferrari-Schlüssel in die Hand gegeben, was zu einem in Europa einzigartig hohen Schutzniveau führen dürfte.«

Die Zahl der Klagen dürfte steigen, »wenn auch wohl nicht dramatisch«, meint Henning (2011). Künftig würden auch kleinere und mittelgroße umweltrelevante Projekte beklagt werden. Aufwändigere Verfahren könnten jedoch dazu beitragen, die Akzeptanz den unmittelbar betroffenen Nachbarn der Großprojekte durch bessere Nachvollziehbarkeit zu stärken. Die Ausweitung der Klagerechte »könnte zwar die Energiewende verschleppen - dürfte aber vielen Wutbürgern den Wind aus den Segeln nehmen «, analysiert ebenso Fischer (2011). Er meint: »Die durchweg positiven Reaktionen aus den Umweltministerien der Länder und aus Berlin auf das Urteil offenbaren, dass darin auch Chancen stecken. Denn durch die Klageberechtigung der Umweltverbände könnte diese Prozessseite endlich 
eine gewisse Berechenbarkeit und höhere Glaubwürdigkeit bekommen. [...] [So] kann damit die Abwägung zwischen Umweltbeeinträchtigungen und Nutzen eines Bauprojekts vor den Augen der Öffentlichkeit stattfinden. Das mag zu mehr Klagen gegen Großprojekte führen, könnte jedoch dafür sorgen, dass nach dem Abschluss eines Prozesses auch die öffentlichen Proteste enden.« (Fischer 2011)

Rechtlich sind die meisten Beschränkungen für die Verbandsklage aus dem Feld geräumt. Das erlaubt die glaubwürdige Drohung mit der Ultima Ratio als äußerstem (nicht unbedingt letztem) Mittel. Das zwingt Projektträger und Behörden, am Verhandlungstisch zu bleiben. Verbandsklagen sind eine Machtdemonstration. Sie haben Signal- und Öffentlichkeitswirkung, auch für Mitglieder und Spender. Für betroffene Projektgegner werden die Verbände als Partner attraktiver, stellt Hofmann (2011) heraus: »Insgesamt wird die Stellung der Umweltverbände erheblich an Bedeutung gewinnen: Vorhabengegner können die Umweltverbände zwischenschalten, um ihre eigenen Rechtsschutzziele zu erreichen.«

Jedoch stößt der Einsatz der Verbandsklage auf praktische Grenzen. Umweltverbände leben von ihrer Reputation. Als verantwortungslose Blockierer dürfen sie nicht dastehen. Wer überreizt, gefährdet die eigene Glaubwürdigkeit und riskiert Kollateralschäden. Im Kontext der Energiewende ist sorgfältig abzuwägen: »[D]ie Verbände müssen nun ihr Selbstverständnis auf den Prüfstand stellen. Es ist eine Sache, nötige Umweltverträglichkeitsprüfungen und Gutachten einzufordern. Eine andere ist es, damit gezielt Bauprojekte verhindern zu wollen. Die Verbände müssen sich nicht auf eine Position zurückziehen, die jeden Eingriff in die Natur ablehnt. Das mag so manchem Molch zunächst nutzen, schadet aber letztlich dem Klima und der Umwelt insgesamt. Wer die Energiewende will, darf den Neubau von Starkstromtrassen, Windrädern und Biomasseanlagen nicht verhindern.«(FTD 2011)

Gut vorbereitete Verbandsklagen haben bisher hohe Erfolgsaussichten, aber keine Erfolgsgarantie. Zudem sind sie teuer. Prozesse dauern oft Jahre. Sie binden viel Personal. Zu tragen sind Anwalts- und Gutachterhonorare, Gerichtskosten und das Risiko von Schadenersatzforderungen, z. B. bei Bauverzögerungen. Für Harald Kächele, Vorsitzender der Deutschen Umwelthilfe und ehemaliger BUND-Landeschef in Berlin, ist Umsicht nötig: »Wir gewinnen meistens, aber wir klagen sehr selten, nur wenn die Risiken begrenzt sind und die
Chance zu gewinnen groß ist. Wir haben die Chance, Großunternehmen richtig weh zu tun. Die Verzögerung bei Projekten kann bei unseren Gegnern extrem ins Geld gehen. Aber davon haben wir unsere eigenen Anwälte noch nicht finanziert. (WIT / DIPA 2005)

So darf nicht überraschen, dass bei Klagen die Tendenz zum Vergleich geht. So geschah es beim Bau der Nord Stream-Gaspipeline durch die Ostsee, beim Offshore-Windpark Nordergründe vor Wangerooge und beim Ausbau des Flughafens Lübeck (NDR Fernsehen 2011). Im Fall der Pipeline zogen die Verbände BUND und WWF ihre Klagen zurück, weil Nord Stream eine Naturschutzstiftung Deutsche Ostsee gründete und mit zehn Millionen Euro Kapital ausstattete. Im Vorstand und Beirat sind - neben Vertretern der Landesregierung Mecklenburg-Vorpommern - BUND, NABU und WWF vertreten. Im Fall Nordergründe zog der BUND eine Klage zurück, nachdem der Betreiber 810.000 Euro an die BUND-nahe Stiftung Naturlandschaft zu spenden bereit war. In Lübeck verzichteten BUND und NABU auf ihre Klage gegen den Flughafenausbau, nachdem der Flughafenbetreiber 2,5 Millionen Euro für die Stiftung Grönauer Heide zugesagt hatte (NDR Fernsehen 2011).

Diese Strategie ist umstritten. Kritiker sehen in diesen Kompromissen einen »Ablasshandel « und sind der Meinung: »Es geht ums Geld und es geht darum, dass man mit großen Firmen und der Politik im stillen Kämmerlein kungelt« (NDR Fernsehen 2011). Der Pipeline-Deal führte u. a. zu heftigem Streit im Landtag MecklenburgVorpommern. Die CDU warf den Umweltverbänden »Käuflichkeit « vor: »Vielfach werden kleinere Maßnahmen, mit denen eine wirkliche Not von Bürgern behoben werden kann, durch das Verbandsklagerecht der Umweltorganisationen jahrelang behindert. Aber bei so großen Investitionen wie der Pipeline gibt es offensichtlich dann ganz andere Sichtweisen.« (Schneider 2011)

Die Verbände wiesen die Angriffe von Medien und Politik scharf zurück. Zum Pipeline-Deal sagte der BUND-Landesverband, er habe sich für eine Eingriffsverringerung und einen angemessenen Ausgleich eingesetzt. Eine Verhinderung des Projektes sei nie das Ziel gewesen. Anlass der Klage sei »eine skandalöse Genehmigung « der Behörden gewesen. Da die gerichtliche Überprüfung eine einjährige Verzögerung des Baustarts bedeutet habe, seien die Investoren an BUND und WWF herangetreten. Ausgleichsmaßnahmen an der Ostsee im Wert von mehr als 20 Millionen Euro seien ermöglicht worden. Nach diesen vertraglichen Zusagen 
seien die naturschutzfachlichen Ziele erreicht gewesen, so dass die Klage zurückgezogen werden konnte (BUND 2011).

\section{Konklusion}

Die Energiewende wird für Vorhabenträger politisch kein Spaziergang. Mehr denn je kommt es für die Projektmanager auf ein integriertes, strategisch und auf Dauer angelegtes Risiko- und Stakeholdermanagement an. Sie werden das latente Protestpotenzial ebenso berücksichtigen müssen wie die bereits organisierte Opposition. Um Akzeptanz werden sie in der Öffentlichkeit werben und kämpfen müssen. Das gilt für regenerative und konventionelle Kraftwerke ebenso wie für die Netze. Die höchstrichterliche Stärkung der Verbandsklagerechte bedeutet die Pflicht, auf Augenhöhe mit den Umweltverbänden zu verhandeln. Beschleunigungsgesetze alter Spielart würden gegen den Widerstand von Bürgerinitiativen und Umweltverbänden nichts beschleunigen, eher ließen sie Konflikte eskalieren. Vom Gesetzgeber ist, wie das Beispiel des NABEG zeigt, trotz des hohen Zeitdrucks für die Energiewende, keine einseitige Bevorzugung der Projektträger zu erwarten. Die Repositionierung des BDI zeigt ebenso: Der Schatten von Stuttgart21 ist lang. Alle Zeichen stehen auf mehr und frühere Bürger- und Verbändebeteiligung sowie mehr Transparenz.

Die Energiewende wird weder durch Bürgerinitiativen noch durch Umweltverbände fundamental bedroht. Sie stellen Forderungen, die schwer zu umgehen, wahrscheinlich teuer und aufwändig sind. In den meisten Fällen geht es jedoch um Anpassungen, nicht um Totalopposition. Eine Ausnahme stellen Kohlekraftwerke dar, die als »Klimakiller « dargestellt werden. Hier ist Ideologisierung zu beobachten, die Verhandlungslösungen kaum Spielraum lässt. Das instrumentelle Spektrum potenzieller Projektgegner zu unterschätzen, wäre ein Fehler. Eine Blockadewelle und Klageflut ist dagegen nicht zu erwarten. Dies erklärt sich sowohl durch eingeschränkte Ressourcen als auch durch die strategische Abwägung von Risiken, Glaubwürdigkeitsund Legitimitätsproblematik, Selbstverständnis und Strategie. Auch für sie sind Kooperation und Verhandlung oftmals die bessere Alternative.

\section{Literatur}

Appel, M. (2011): Umweltverbände im Ferrari des deutschen Umweltrechtsschutzes - Anmerkung zur Trianel-Entscheidung des EuGH, Urt. v. 12.5.2011 - C-115/09. Natur und Recht, 33 (6), 414-416.

BDI (2011a): Investitionsprojekte vorantreiben, http://www.bauindustrie.de/media/attachments/BDI-InvestitionsAgenda_27_ Juni_2011_small.pdf, Zugriff: 14.09.2011.

BDI (2011b): Lange Verfahren hemmen Investitionen, http://www. investitionsagenda.de/node/306/145, Zugriff: 13.09.2011.

Becké, A. B. (2011): »Wutbürger?«, http://www.demokratie-goettingen.de/blog/\%E2\%80\%9Ewutburger\%E2\%80\%9C/, Zugriff: 16.09.2011.

Becker, J. (2011): Vom Wutbürger zum Mutbürger: Umweltverband fordert stärkere Beteiligung der Öffentlichkeit bei Großprojekten, http://www.neues-deutschland.de/artikel/187799.vom-wutbuerger-zum-mutbuerger.html, Zugriff: 20.05.2011.

Bernard, A., Heidtmann, J., Luef, W. (2011): Gut, dass Sie da sind! Süddeutsche Zeitung Magazin (13), 20-30.

Börnecke, S. (2003): Gekämpft wird diesmal anders - auf beiden Seiten. Frankfurter Rundschau, 35.

BUND (2011a): Erklärung in eigener Sache: Der BUND ist und bleibt unbestechlich!, http://www.bund-mecklenburg-vorpommern.de/ themen_und_projekte/ostseeschutz/ostseepipeline/erklaerung/, Zugriff: 05.09.2011.

BUND (2011b): Mehr Bürger- und Klagerechte gegen Großprojekte: EuGH-Entscheidung zum Kohlekraftwerk Lünen, http:// www.bund.net/fileadmin/bundnet/pdfs/klima_und_energie/20110512_klima_energie_hintergrund_eugh_luenen.pdf, Zugriff: 02.09.2011.

Bundesregierung (2011): Grünes Licht für Ausbau der Stromnetze, http://www.bundesregierung.de/Content/DE/ Artikel/2011/06/2011-06-06-energiewende-ausbau-stromnetze. html, Zugriff: 13.09.2011.

Claus, F., Dames, S. (2011): Industriepolitik, http://www.dialoggestalter.de/themen/aktuelle-themen/industriepolitik.html, Zugriff: 03.09.2011.

EPAW (2011): Über uns, http://epaw.org/about_us.php?lang=de, Zugriff: 10.09.2011.

Financial Times Deutschland (2011): Klägliches Verfahren. Financial Times Deutschland, 1.

Fischer, K. (2011): Anklage im Namen der Fledermaus, http://www. wiwo.de/politik-weltwirtschaft/anklage-im-namen-der-fledermaus-466200/, Zugriff: 02.09.2011.

Glaberson, W. (1988): Coping in the Age of ,Nimby', http://www. nytimes.com/1988/06/19/business/coping-in-the-age-of-nimby. html, Zugriff: 02.09.2011.

Henning, B. (2011): Erweiterung der Klagerechte anerkannter Umweltverbände - Chance auf mehr Umweltschutz oder Investitionshindernis? Neue Juristische Wochenschrift NJW (38), 2765-2769.

Hofmann, H. (2011): Höhere Hürden für industrielle Großanlagen. Energie \& Management.

Hörr, S. (2011): Engagement, bevor der Wutbürger eingreift, http:// www.berlinonline.de/berliner-zeitung/archiv/.bin/dump. fcgi/2011/0603/sonderthema/0022/index.html, Zugriff: 02.09.2011.

Inhaber, H. (1998): Slaying the NIMBY dragon. Transaction, New Brunswick und London. 
Klima-Allianz Deutschland (2010): Wer wir sind: Aktivitäten, http:// www.die-klima-allianz.de/wer-wir-sind/aktivitaten, Zugriff: 04.09.2011.

Klima-Allianz Deutschland (2011): Erfolge für Kohlekraftwerksgegner, http://kohle-protest.de/erfolge0/, Zugriff: 11.09.2011.

Koch, H.-J. (2007): Die Verbandsklage im Umweltrecht. Neue Zeitschrift für Verwaltungsrecht NVwZ (4), 369-379.

Kreutzfeldt, M. (2010): Strategie der Klima-Allianz: Lobbyismus statt Aktionismus, http://www.taz.de/!49421/, Zugriff: 05.09.2011.

Kurbjuweit, D. (2011): Der Wutbürger. Der Spiegel (41), 26-27.

Mahnke, E. (2011): Energiewende-Gesetze: Networking beschleunigt, http://www.klimaretter.info/politik/gesetze-zurenergiewende/9259-gesetze-zur-energiewende-networkingbeschleunigt, Zugriff: 13.09.2011.

Merkel, A. (2011): Der Weg zur Energie der Zukunft, Regierungserklärung, Plenarprotokoll 17/114, http://dipbt.bundestag.de/dip21/ btp/17/17114.pdf\#P.12958, Zugriff: 02.09.2011.

Müller, S., Hofmann, H. (2011): EuGH weitet Klagerecht von Umweltverbänden deutlich aus, http://www.oppenhoff.eu/presse/newsletter/article/newsletter-umweltrecht-mai-2011.html, Zugriff: 20.08.2011

NABU (2009): Netzausbau und -anpassung zur Integration Erneuerbarer Energiequellen - NABU-Grundsätze, http://www.nabu.de/ imperia/md/content/nabude/energie/nabu-grunds_tze_netzausbau_090401.pdf, Zugriff: 09.11.2011.

NDR Fernsehen (2011): Tauschgeschäfte - Umweltverbände verraten Ideale, http://www.ndr.de/fernsehen/sendungen/menschen_und_schlagzeilen/umweltverbaende101.html, Zugriff: 02.09.2011.

Rath, C. (2006): »Klarer Verstoß gegen EU-Recht«. taz, die Tageszeitung, 6 .

Remmel, J. (2006): Umfassende »Verbandsklage« heißt: Naturschutz auf gleicher Augenhöhe! Die Grünen im Landtag NRW, Zugriff: 15.05.2011.

Saint, M., Flavell, R. J., Fox, P. F. (2009): Nimby wars: the politics of land use. Saint University Press, Hingham, Mass.

Schlacke, S. (2004): Rechtsschutz durch Verbandsklage: Zum Fortentwicklungsbedarf des umweltbezogenen Rechtsschutzsystems. Natur und Recht, 26 (10), 629-635.

Schmidt, A. (2008): Verbandsklagen im Naturschutzrecht und Realisierung von Infrastrukturvorhaben - Ergebnisse einer empirischen Untersuchung. Natur und Recht, 30 (8), 544-553.

Schneider, M. (2011): Harter Vorwurf gegen Umweltverbände, http:// www.welt.de/print/die_welt/wirtschaft/article12872113/HarterVorwurf-gegen-Umweltverbaende.html, Zugriff: 05.09.2011.

Schoppen, C. (2011): EuGH stärkt Klagerechte der Umweltverbände, http://www.luther-lawfirm.com/download_aktuelles_de/220.pdf, Zugriff: 02.09.2011.

Shah, T., Karten, I. (2011): Wege aus der »Dagegen-Falle«. Politik \& Kommunikation, 42-43.

Sharpston, E. (2011): Beteiligung der Öffentlichkeit an bestimmten umweltbezogenen Plänen und Programmen: Schlussanträge der Generalanwältin Eleanor Sharpston vom 16.12.2010. In: Rechtssache C-115/09. Natur und Recht, 33 (1), 72-80.

Thomsett, M. C. (2004): Nimbyism: navigating the poitiics of local opposition. Center Line, Arlington, Va.

Unmüßig, B. (2011): NGOs in der Klimakrise: Fragmentierungsprozesse, Konfliktlinien und strategische Ansätze, http://www.boell. de/oekologie/gesellschaft/oekologie-gesellschaft-ngos-in-derklimakrise-11001.html, Zugriff: 01.11.2011. von Daniels, G., Uibeleisen, M. (2011): Die Kehrseite des TurboAusstiegs aus der Kernkraft, http://www.ftd.de/politik/ deutschland/:deutscher-sonderweg-die-kehrseite-des-turbo-ausstiegs-aus-der-kernkraft/60037743.html, Zugriff: 13.09.2011.

Walter, F. (2011): Studie über »Wutbürger«: Alt, stur, egoistisch, http:// www.spiegel.de/politik/deutschland/0,1518,784664,00.html, Zugriff: 08.09.2011.

Wegener, B. (2011): Die europäische Umweltverbandsklage. Zeitschrift für Umweltrecht ZUR (7-8), 363-368.

Wirner, S. (2011): »Die Leute waren zu Recht wütend«, http://www. drehscheibe.org/interview-mit-heiner-geissler.html, Zugriff: 03.09.2011.

WIT/DIPA (2005): Bewahren, blockieren, bauen lassen? Großprojekte der Wirtschaft zwischen Verhandlungs- und Verhinderungs-Demokratie, http://www.marcoalthaus.de/resources/ Bericht+Grossprojekte+Veto-Demokr.pdf, Zugriff: 05.09.2011.

Autor

\section{Prof. Dr. Marco Althaus}

Sozialwissenschaften

Fachbereich Wirtschaft, Verwaltung und Recht

Technische Hochschule Wildau [FH]

$\mathrm{T}+493375$ 508-341

marco.althaus@th-wildau.de 